\title{
Ölçülen Yapraklanmaların Genelleștirilmiş Dynnikov Koordinat Sistemi
}

\author{
Alev Meral Dülger \\ Dicle Üniversitesi, Fen Fakültesi, Matematik Bölümü, Diyarbakır, Türkiye \\ ve \\ Orta Doğu Teknik Üniversitesi, Fen Edebiyat Fakültesi, Matematik Bölümü, Ankara, Türkiye, (ORCID: 0000-0002-9838-7906), alev.meral@ dicle.edu.tr \\ (1st International Conference on Applied Engineering and Natural Sciences ICAENS 2021, November 1-3, 2021)
}

(DOI: 10.31590 /ejosat.)

\begin{abstract}
ATIF/REFERENCE: Meral Dülger, A. (2021). Ölçülen Yapraklanmaların Genelleştirilmiş Dynnikov Koordinat Sistemi. Avrupa Bilim ve Teknoloji Dergisi, (28), 437-440.

$\ddot{O} z$

Bu çalışmada, $n(n \geq 2)$ adet işaretlenmiş nokta ve 1 adet sınır bileşenine sahip olan, 1 cinsli yönlendirilebilir $S_{n}$ yüzeyindeki integral laminasyonlar için elde edilen genelleştirilmiş Dynnikov koordinatları, aynı yüzeyde tanımlı ölçülen yapraklanmalara genişletilmiştir. Daha açık olarak, $S_{n}$ yüzeyinde tanımlı ölçülen yapraklanmaların uzayı ve $V_{n}=\{(a ; b ; T ; c): c \leq 0$ ve $\mathrm{T} \neq 0\} \cup\{0\}$ olmak üzere $\mathbb{R}^{2 n+2} \backslash V_{n}$ arasında bir homeomorfizm tanımlayan genelleştirilmiş Dynnikov koordinat sistemi tanıtılmıştır.
\end{abstract}

Anahtar Kelimeler: Ölçülen yapraklanma, İntegral laminasyon, Genelleştirilmiş Dynnikov koordinatları, Geometrik kesişim sayısı, İşaretlenmiş noktalı torus.

\section{Generalized Dynnikov Coordinate System of Measured Foliations}

\begin{abstract}
In this paper, the generalized Dynnikov coordinates obtained for the integral laminations on an orientable surface $S_{n}$ of genus 1 with $n(n \geq 2)$ punctures and 1 boundary component are extended to the measured foliations defined on the same surface. More specifically, the generalized Dynnikov coordinate system, which defines a homeomorphism between the space of measured foliations defined on $S_{n}$ and $\mathbb{R}^{2 n+2} \backslash V_{n}$, where $V_{n}=\{(a ; b ; T ; c): c \leq 0$ ve $\mathrm{T} \neq 0\} \cup\{0\}$, is introduced.
\end{abstract}

Keywords: Measured foliation, Integral lamination, Generalized Dynnikov coordinates, Geometric intersection number, Punctured torus. 


\section{Giriş}

Bu çalışmanın amacı, $n(n \geq 2)$ adet işaretlenmiş nokta ve 1 adet sınır bileşenine sahip olan, 1 cinsli yönlendirilebilir $S_{n}$ yüzeyindeki integral laminasyonlar (bir integral laminasyon, sonlu sayıda esas basit kapalı eğrinin izotopi sınıflarının ayrık bir birleşimidir) için elde edilen genelleştirilmiş Dynnikov koordinatlarını, yine aynı yüzeydeki ölçülen yapraklanmalar için genişletmektir. Yüzeyin sonlu adet işaretlenmiş noktalı $D_{n}(n \geq$ 3) diski olmas1 durumunda, integral laminasyonları koordinatlandırmanın etkili bir yolu Dynnikov koordinat sistemini kullanmaktır (Dynnikov, 2002). 2002 y1lında Dynnikov tarafından tanıtılan Dynnikov koordinatları ve $n$-örgü grubunun Dynnikov koordinatları cinsinden etkisini veren kurallar bir çok dinamiksel ve kombinatorik problemin çözümünde kullanılmıştır. Dynnikov koordinatları, (Dehornoy, 2008) ve (Dehornoy ve ark., 2002)'de $n$-örgü grubunda kelime probleminin çözümünde çalışılmıştır. Daha sonra Moussafir örgülerin topolojik entropilerini yaklaşık olarak hesaplayan bir metot tanıtmıştır (Moussafir, 2006). Yurttaş, Moussafir'in tekniğinden esinlenerek Dynnikov koordinatları yardımıyla pseudo-Anosov örgü ailelerinin topolojik entropisini hesaplayan bir metot geliştirmiştir (Yurttaş, 2011). Meral, sonlu sayıda işaretlenmiş noktalı disk yüzeyindeki integral laminasyonlar için tanımlı Dynnikov koordinat sistemini, 1 adet sınır bileşeni ve $n(n \geq 2)$ adet işaretlenmiş noktası olan 1 cinsli $S_{n}$ yüzeyine genelleştirmiştir (Meral, 2021). Bu çalışmada, genelleştirilmiş Dynnikov koordinat sisteminin sebep olduğu, $S_{n}$ 'deki integral laminasyonların kümesi ile $V_{n}=\{(a ; b ; T ; c): c \leq 0$ ve $\mathrm{T} \neq$ $0\} \cup\{0\}$ olmak üzere $\mathbb{Z}^{2 n+2} \backslash V_{n}$ arasındaki birebir ve örten dönüşüm, $S_{n}$ 'deki ölçülen yapraklanmaların uzayı ile $\mathbb{R}^{2 n+2} \backslash V_{n}$ arasındaki homeomorfizmaya genişletilecektir. Bunun için, $S_{n}$ yüzeyine gömülü $3 n+2$ adet yay ve 1 adet kapalı eğriden oluşan bir system kullanılmıştır. Verilen bir $F$ ölçülen yapraklanması, bu sistemdeki yaylar ve eğri üzerine tayin edilen ölçüler kullanılarak $\mathbb{R}_{\geq 0}^{3 n+3} \backslash\{0\}$ kümesinin bir elemanı tarafından tanımlanmıştır. $S_{n}$ 'de tanımlı ölçülen yapraklanmaların kümesi $\operatorname{MF}\left(S_{n}\right)$ olsun. Genelleştirilmiş Dynnikov koordinatları, yukarıda bahsedilen ölçülerin bir lineer bileşimidir. Genelleştirilmiş Dynnikov koordinat fonksiyonu, $\operatorname{MF}\left(S_{n}\right)$ uzayı ile $\mathbb{R}^{2 n+2} \backslash V_{n}$ arasinda bir homeomorfizma vermektedir.

\section{Materyal ve Yöntem}

$\mathrm{Bu}$ bölümde, $S_{n}$ 'deki genelleştirilmiş Dynnikov koordinatlarını tanımlayacağız. Bunun için, Şekil 1'de gösterilen modeli kullanacağız. Burada $\alpha_{i}(1 \leq i \leq 2 n)$ ve $\beta_{i}(1 \leq i \leq$ $n+1)$ yayları, işaretlenmiş noktalı disk durumuna benzerdir. Böylece, bu yayların uç noktaları yüzeyin sınırında velveya işaretlenmiş noktaların üzerindedir. $c$, cinsi bir defa saran kapalı eğri ve $\gamma$, her iki uç noktası yüzeyin sınırında olan yaydır.

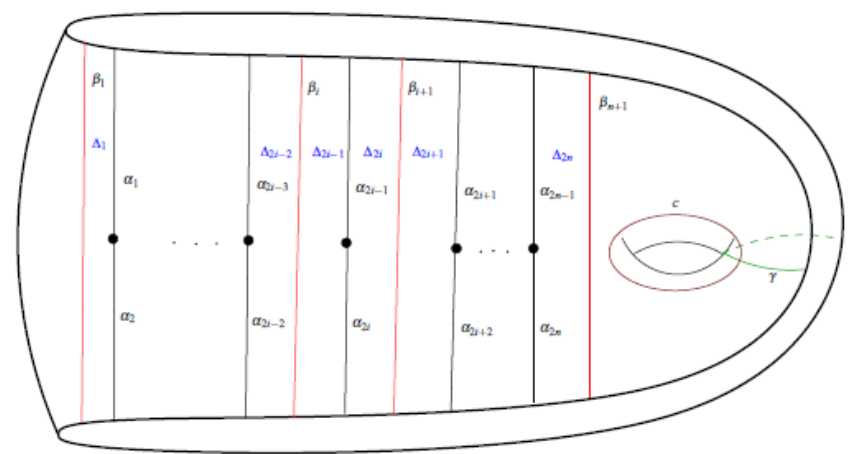

Şekil 1. $\alpha_{i}, \beta_{i}, \gamma_{i}$ yayları ve $c$ eğrisi (Meral, 2021)

$S_{n}$ 'deki integral laminasyonların kümesi (bir integral laminasyon, uygun izotopi altında ayrık esas basit kapalı eğrilerin sonlu bir birleşimidir; örneğin, Şekil 2'ye bakabilirsiniz) $L_{n}$ ve $L \in L_{n}$ olsun. Çalışma boyunca $L$ 'nin ve $F$ 'nin minimal birer temsilcileriyle çalışılmaktadır ve bu temsilciler sırasıyla $l$ ve $f$ (koordinat eğrilerini minimal bir şekilde kesen (ölçülen yapraklanma için, minimal bir şekilde ölçen) aynı izotopi sınıfındaki bir integral laminasyondur (bir ölçülen yapraklanmadır, sırasıyla)) ile gösterilmektedir. $\left(\alpha_{1}, \ldots, \alpha_{2 n} ; \beta_{1}, \ldots, \beta_{n+1} ; \gamma ; c\right) \in\left\{\mathbb{Z}_{\geq 0}^{3 n+3}\right\} \backslash\{0\} \quad$ vektörü, l'nin karşılık gelen yaylar ve $c$ basit kapalı eğrisiyle olan kesişim sayılarını göstersin. Örneğin $(4,1,3,2,4,1 ; 3,5,5,3 ; 3 ; 1)$, Şekil 2 'de resmedilen $l$ integral laminasyonunun kesişim sayılarıdır.

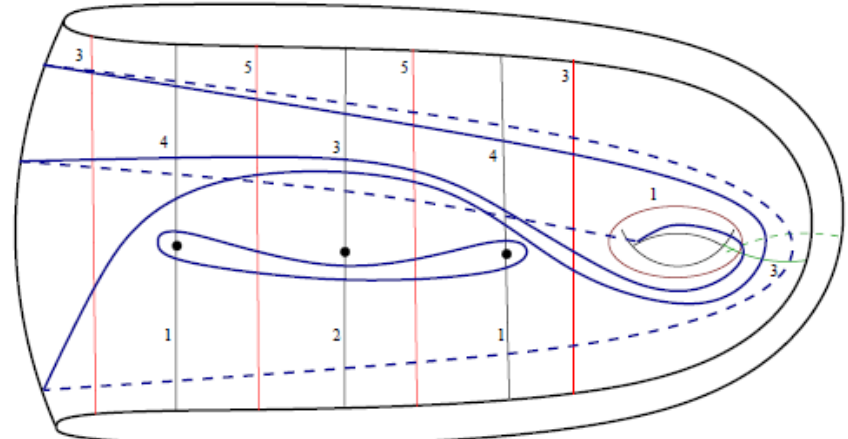

Şekil 2. İntegral laminasyon l'nin koordinat eğrileriyle kesişim sayıları (Meral, 2021)

Tanım 1. $V_{n}=\{(a ; b ; T ; c): c \leq 0$ ve $\mathrm{T} \neq 0\} \cup\{0\}$ olsun. Genelleştirilmiş Dynnikov koordinat fonksiyonu $\Theta: L_{n} \rightarrow$ $\mathbb{Z}^{2 n+2} \backslash V_{n}$ her $1 \leq i \leq n$ için

$$
a_{i}=\frac{\alpha_{2 i}-\alpha_{2 i-1}-c^{+}}{2}, \quad b_{i}=\frac{\beta_{i}-\beta_{i+1}}{2}
$$

ve

$$
|T|= \begin{cases}0, & \text { ĕger } c^{+}=0, \\ \gamma-\frac{\beta_{n+1}-c^{+}}{2}-\frac{\beta_{1}-c^{+}}{2}, & \text { ĕger } c^{+} \neq 0,\end{cases}
$$

olmak üzere,

$$
\Theta(L)=(a ; b ; T ; c)=\left(a_{1}, \ldots, a_{n} ; b_{1}, \ldots, b_{n} ; T ; c\right)
$$

şeklinde tanımlanır. Kesen bileşenler pozitif yönde burgu yaptığında $\operatorname{sgn}(T)=+1$, negatif yönde burgu yaptığında $\operatorname{sgn}(T)=-1$ 'dir (sgn, işaret (signum) fonksiyonudur).

Aşağıdaki teorem genelleştirilmiş Dynnikov koordinat fonksiyonunun tersini vermektedir. İspatını (Meral, 2021)'de bulabilirsiniz. 
Teorem 1. $(a ; b ; T ; c) \in \mathbb{Z}^{2 n+2} \backslash V_{n} \quad$ olsun. O zaman $(a ; b ; T ; c)$ vektörü, geometrik kesişim sayıları aşağıda verilen bir ve yalnız bir $L \in L_{n}$ integral laminasyonuna karşılık gelir.

$$
\kappa=\max _{1 \leq k \leq n}\left[2 \max \left(b_{k}, 0\right)+\left|2 a_{k}+c^{+}\right|-2 \sum_{j=k}^{n} b_{j}\right]
$$

olmak üzere

$$
\begin{aligned}
& \beta_{i}=2 \sum_{j=i}^{n} b_{j}+\max \left(c^{+}, c^{+}-2 \sum_{i=1}^{n} b_{i}, \kappa\right), \\
& \beta_{n+1}=\max \left(c^{+}, c^{+}-2 \sum_{i=1}^{n} b_{i}, \kappa\right)
\end{aligned}
$$

$\alpha_{i}= \begin{cases}\frac{2(-1)^{i} a_{[i / 2]}+(-1)^{i} c^{+}+\beta_{[i / 2]}}{2}, & \text { ĕger } b_{[i / 2]} \geq 0, \\ \frac{2(-1)^{i} a_{[i / 2]}+(-1)^{i} c^{+}+\beta_{(1+[i / 2]}}{2}, & \text { ĕger } b_{[i / 2]} \leq 0,\end{cases}$

ve

$$
\gamma= \begin{cases}|T|+\sum_{j=1}^{n} b_{j}+\max \left(c^{+}, c^{+}-2 \sum_{i=1}^{n} b_{i}, \kappa\right)-c^{+}, & \text {ĕger } c>0, \\ |c|+\sum_{j=1}^{n} b_{j}+\max \left(c^{+}, c^{+}-2 \sum_{i=1}^{n} b_{i}, \kappa\right)-c^{+}, & \text {ĕger } c \leq 0 .\end{cases}
$$

Burada $\lceil x\rceil, x$ 'den küçük olmayan en küçük tam sayıdır. Kesen bileşenler pozitif yönde burgu yaptığında $\operatorname{sgn}(T)=+1$, negatif yönde burgu yaptığında $\operatorname{sgn}(T)=-1$ 'dir (sgn, işaret (signum) fonksiyonudur).

\section{1. Ölçülen Yapraklanmalar}

Bu bölümde ölçülen yapraklanma konusu için gerekli olan genel bilgiler verilecektir.

Tanım 2. $S_{n}$ 'de bir $F$ tekil yapraklanması, $S_{n}$ 'nin yaprak adı verilen birbirinden ayrık yol bağlantılı alt kümelerine bir parçalanışıdır ve aşağıdaki koşulları sağlar:

i. Başlangıcı tekil nokta olan yaprak parçalarına çatal denir. Tekil noktalar, $p \geq 1$ çatal sayılarına gore sınıflandırılır. $S_{n}^{o}$ 'deki bir $p$-çatallı tekil nokta civarındaki harita lokal olarak Şekil 3'teki gibidir. 1-çatallı tekil noktalar sadece $S_{n}$ 'nin işaretlenmiş noktalarında olabilir.
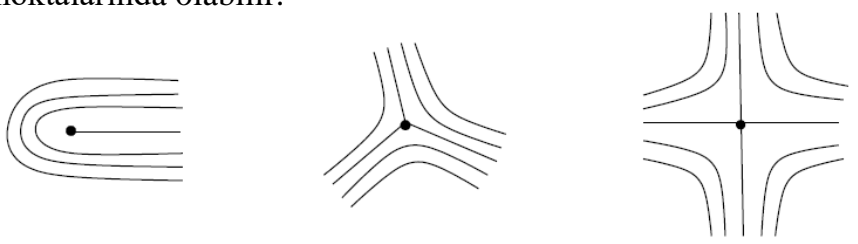

Şekil 3. $S_{n}^{o}$ 'deki yapraklanmaların 1-çatallı, 3-çatallı, 4-çatallı tekil noktaları

ii. Sonlu bir $J$ tekil nokta kümesi dışındaki her $x \in S_{n} \backslash \partial S_{n}$ noktası Şekil 4'de gösterildiği gibi, $U \cap$ yaprak bileșenlerini yatay doğrulara taşıyan bir $\phi: U \rightarrow \mathbb{R}^{2}$ haritası tarafından kapsanir.

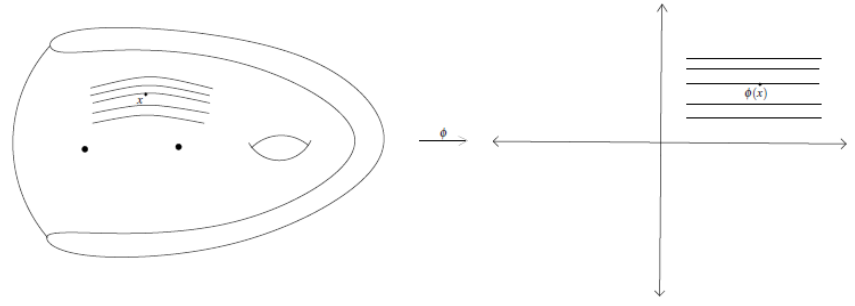

Şekil 4. $x \in S_{n} \backslash \partial S_{n}$ yakınındaki yapraklar

Tanım 3. $S_{n}$ 'de bir $\alpha$ yayı, Şekil 5 'de gösterildiği gibi $F$ tekil yapraklanmasının tüm yapraklarını çapraz bir şekilde kessin ve bir $s \in J$ tekil noktasına girdiği bölge çıktığı bölgeden farklı olsun. Bu durumda $\alpha$ yayı $F$ 'ye çaprazdır denir.

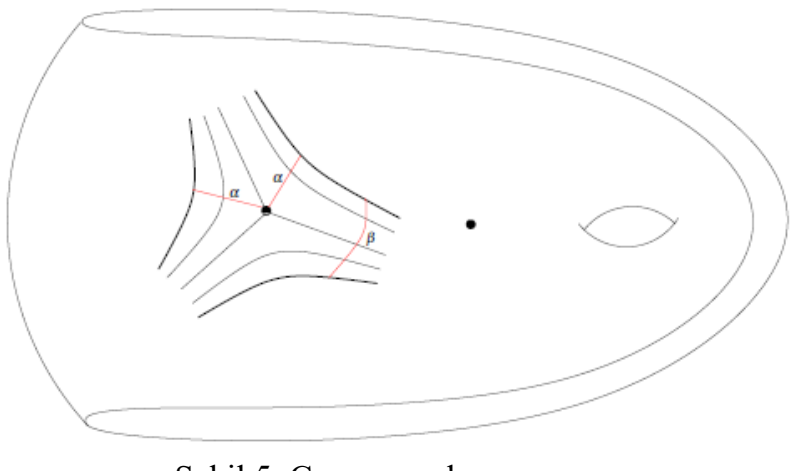

Şekil 5. Çapraz yaylar

Tanım 4. Bir $F$ yapraklanması verildiğinde, $F$ üzerindeki bir çapraz ölçüm $\mu$, her çapraz $\alpha$ yayına bir $\mu(\alpha) \in \mathbb{R}^{+}$pozitif sayısı atayan ve Şekil 6'da resmedilen aşağıdaki özellikleri sağlayan bir fonksiyondur:
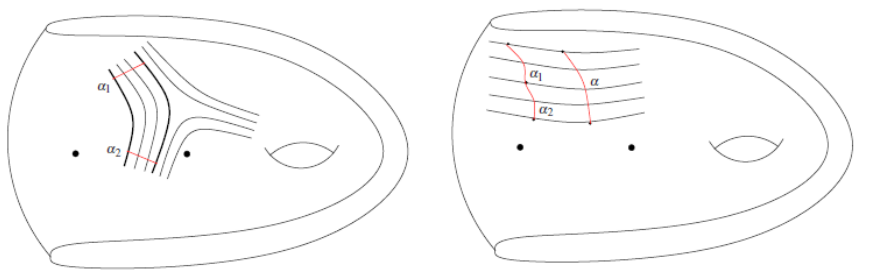

Şekil 6. $\mu\left(\alpha_{1}\right)=\mu\left(\alpha_{2}\right)$ ve $\mu\left(\alpha_{1}\right)+\mu\left(\alpha_{2}\right)=\mu(\alpha)$

i. $\alpha_{1}$ ve $\alpha_{2}$ çapraz yayları, uç noktaları aynı yapraklar üzerinde olan diğer çapraz yaylar aracılığı ile birbirine izotop ise $\mu\left(\alpha_{1}\right)=\mu\left(\alpha_{2}\right)^{\prime}$ dir.

ii. $\alpha_{1} \cap \alpha_{2}=\partial \alpha_{1} \cap \partial \alpha_{2}$ olacak şekilde $\alpha=\alpha_{1} \cup \alpha_{2}$ ise $\mu(\alpha)=\mu\left(\alpha_{1}\right)+\mu\left(\alpha_{2}\right)^{\prime}$ dir.

Tanım 5. $S_{n}$ 'de bir $(F, \mu)$ ölçülen yapraklanması, bir $\mu$ çapraz ölçümü ile donatılmış tekil yapraklanmadır.

Tanım 6. $(F, \mu), S_{n}$ 'de bir ölçülen yapraklanma ve $\alpha, S_{n}$ 'de bir yay olsun. Yapraklanma üzerinde tanımlı $\mu$ ölçümünü kullanarak $\mu(\alpha)$ ölçüsü, $\alpha_{1}, \ldots, \alpha_{k} \quad \alpha^{\prime}$ nın yapraklanmaya çapraz birbirinden ayrık alt yayları olmak üzere

$$
\mu(\alpha)=\sup \sum_{i=1}^{k} \mu\left(\alpha_{i}\right)
$$


olarak tanımlanır. $[\alpha]$, uç noktalara göre alınan izotopiler altında $\alpha$ 'nın izotopi sınıfinı belirtsin. $\beta$ yayları $\alpha$ 'a izotop ve $(F, \mu)$ 'e çapraz olmak üzere

$$
\mu[\alpha]=\inf _{\beta \in[\alpha]} \mu(\beta)
$$

olarak tanımlanır. Benzer şekilde, $C$ elemanter kapalı esas eğrisi ve onun izotopi sınıfı $[C]$ için $\mu(C)$ ve $i(F,[C])$ tanımlanabilir.

\section{Bulgular}

İntegral laminasyonlar için elde edilen genelleştirilmiş Dynnikov koordinatları doğal bir şekilde ölçülen yapraklara genişletilebilir. $\mathrm{Bu}$ bölümde, integral laminasyonlar için elde edilen genelleştirilmiş Dynnikov koordinat sistemi ölçülen yapraklanmalara genişletilecektir.

Ölçülen yapraklanma için genelleştirilmiş Dynnikov koordinat fonksiyonu aşağıdaki gibi tanımlanmıştır:

Tanım 7. $V_{n}=\{(a ; b ; T ; c): c \leq 0$ ve $\mathrm{T} \neq 0\} \cup\{0\}$ olsun. $\vartheta: M F\left(S_{n}\right) \rightarrow \mathbb{R}^{2 n+2} \backslash V_{n}$ genelleştirilmiş Dynnikov koordinat fonksiyonu, her $1 \leq i \leq n$ için

$$
\begin{gathered}
a_{i}=\frac{\mu\left(\left[\alpha_{2 i}\right]\right)-\mu\left(\left[\alpha_{2 i-1}\right]\right)-\mu\left(\left[c^{+}\right]\right)}{2}, \\
b_{i}=\frac{\mu\left(\left[\beta_{i}\right]\right)-\mu\left(\left[\beta_{i+1}\right]\right)}{2}
\end{gathered}
$$

ve

$$
|T|= \begin{cases}0, & \text { ĕ } \operatorname{er} \mu\left(\left[c^{+}\right]\right)=0, \\ \mu([\gamma])-\frac{\mu\left(\left[\beta_{n+1}\right]\right)-\mu\left(\left[c^{+}\right]\right)}{2}-\frac{\mu\left(\left[\beta_{1}\right]\right)-\mu\left(\left[c^{+}\right]\right)}{2}, & \text { ĕg } \operatorname{er~} \mu\left(\left[c^{+}\right]\right) \neq 0,\end{cases}
$$

olmak üzere,

$$
\vartheta(F)=(a ; b ; T ; c)=\left(a_{1}, \ldots, a_{n} ; b_{1}, \ldots, b_{n} ; T ; c\right)
$$

olarak tanımlanır. Kesen bileşenler pozitif yönde burgu yaptığında $\operatorname{sgn}(T)=+1$, negatif yönde burgu yaptı̆̆ında $\operatorname{sgn}(T)=-1$ 'dir (sgn, işaret (signum) fonksiyonudur).

Aşağıdaki teorem, ölçülen yapraklanma için genelleştirilmiş Dynnikov koordinat fonksiyonunun tersini vermektedir. İspat1, Teorem 1'in ispatı ile aynıdır.

Teorem 2. $(a ; b ; T ; c) \in \mathbb{R}^{2 n+2} \backslash V_{n}$ olsun. O zaman $(a ; b ; T ; c)$ vektörü,

$$
\kappa=\max _{1 \leq k \leq n}\left[2 \max \left(b_{k}, 0\right)+\left|2 a_{k}+c^{+}\right|-2 \sum_{j=k}^{n} b_{j}\right]
$$

olmak üzere

$$
\begin{gathered}
\mu\left(\left[\beta_{i}\right]\right)=2 \sum_{j=i}^{n} b_{j}+\max \left(c^{+}, c^{+}-2 \sum_{i=1}^{n} b_{i}, \kappa\right) \\
\mu\left(\left[\beta_{n+1}\right]\right)=\max \left(c^{+}, c^{+}-2 \sum_{i=1}^{n} b_{i}, \kappa\right)
\end{gathered}
$$

$\mu\left(\left[\alpha_{i}\right]\right)= \begin{cases}\frac{2(-1)^{i} a_{[i / 2]}+(-1)^{i} c^{+}+\mu\left(\left[\beta_{[i / 2]}\right]\right)}{2}, & \text { ĕger } b_{[i / 2]} \geq 0, \\ \frac{2(-1)^{i} a_{[i / 2]}+(-1)^{i} c^{+}+\mu\left(\left[\beta_{(1+[i / 2]}\right]\right)}{2}, & \text { ĕger } b_{[i / 2]} \leq 0,\end{cases}$ ve

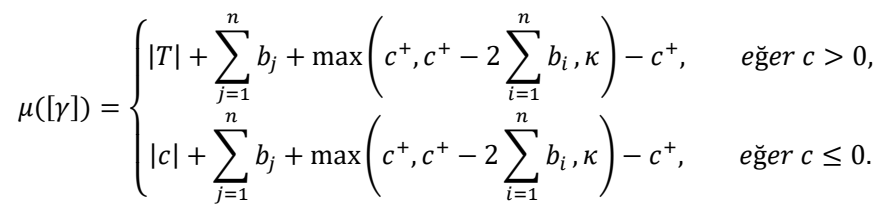

olacak şekilde bir ve yalnız bir $(F, \mu) \in M F\left(S_{n}\right)$ ölçülen yapraklanmasına karşılık gelir. Burada $\lceil x\rceil, \quad x$ 'den küçük olmayan en küçük tam sayıdır. Kesen bileşenler pozitif yönde burgu yaptığında $\operatorname{sgn}(T)=+1$, negatif yönde burgu yaptığında $\operatorname{sgn}(T)=-1$ 'dir.

\section{Sonuç}

$\mathrm{Bu}$ çalışmada, daha önce sadece sonlu bir şekilde işaretlenmiş noktalı disk üzerindeki ölçülen yapraklanmaları tek türlü olarak tanımlayan Dynnikov koordinat sistemi $S_{n}$ yüzeyine genelleştirilmiştir. $S_{n}$ yüzeyindeki ölçülen yapraklanmaları tek türlü koordinatlayan genelleştirilmiş Dynnikov koordinat sistemi ile birçok yeni problem çözülmesi planlanmaktadır. Örneğin, genelleştirilmiş Dynnikov koordinat sisteminin $g>1$ cinsli yüzeylere genelleştirilmesi bunlardan biridir.

NOT: Yazar, bu çalışmadaki sonuçların yazarın doktora tezinden bir parça olduğunu belirtmek istemektedir.

\section{Kaynakça}

Dynnikov, I. (2002). On a Yang-Baxter mapping and the Dehornoy ordering. Uspekhi Mat. Nauk, 57(3(345)), 151152.

Dehornoy, P. (2008). Efficient solutions to the braid isotopy problem. Discrete Appl. Math., 156(16), 3091-3112.

Dehornoy, P., Dynnikov, I., Rolfsen, D., Wiest, B. (2002). Why are braids orderable?. Panoramas et Syntheses [Panoramas and Syntheses]. Societe Mathematique de France, Paris, 14.

Moussafir, J. (2006). On computing the entropy of braids. Funct. Anal. Other Math., 1, 37-46.

Yurttaş, S. Ö. (2011). Dynnikov coordinates and pseudo-Anosov braids. Doktora tezi, Liverpool Üniversitesi Fen Bilimleri Enstitüsü, Liverpool, 168.

Meral, A. (2021). Dynnikov coordinates on punctured torus. Turkish Journal of Mathematics, 45(2), 661-677. 